\title{
JOINT CHANGES IN NEUROFIBROMATOSIS
}

\section{Report of Two Cases}

\section{A. C. Bingold, London, ENglind}

Gould (1918) stated that neurofibromatosis may cause pigmentation of the skin, mental deficiency, changes in the skeleton and changes in the joints. What the joint changes were he failed to specify, nor have they been described by a later writer. I intend therefore to analyse the joint changes in neurofibromatosis, after presenting two illustrative cases.

\section{GASE REPORTS}

Case 1-A thirty-seren-year-old woman complained of intermittent pain in her left knee for the last twenty years, and of constant pain in her left groin for the last seven months. For as long as she could remember she had had lumps in her skin and a limp. She was the roungest of ten children; no other member of her family was similarly affected.

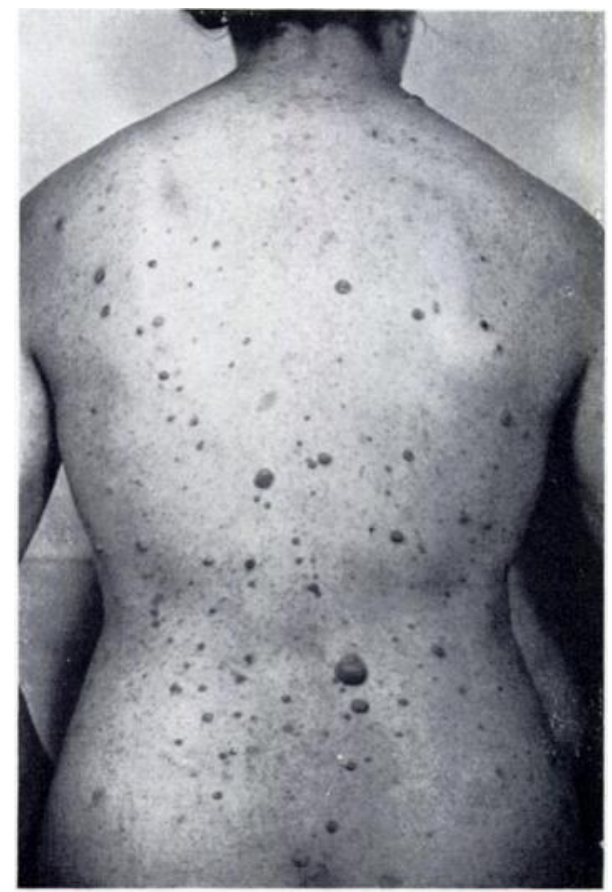

FIG. 1

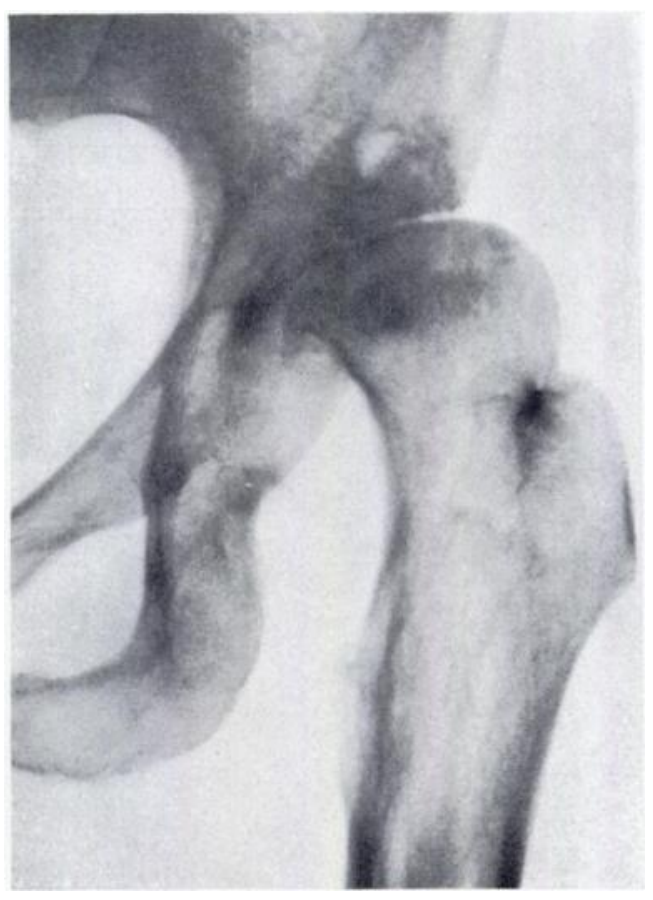

FIG. 2

Case 1. Figure 1-Photograph of the patient's back showing many subcutaneous tumour nodules and a few café au lait areas. Figure 2-Radiograph of left hip showing a shallow dense acetabulum, a translucent area above it, a well marked os acetabuli, diminution of the joint space, a flat, dense, partly subluxated femoral head, and coxa valga.

On examination there were multiple subcutaneous nodules and a few café au lait areas (Fig. 1). A number of mobile, tender, subdermal nodules, one to two centimetres in diameter, were palpable in her left groin and round her left knee. The left femur was five centimetres shorter than the right. She walked with a marked dip to the left, and there was a compensatory lumbar scoliosis. The movements of her hips and knees were full, but the left knee was slightly unstable. 


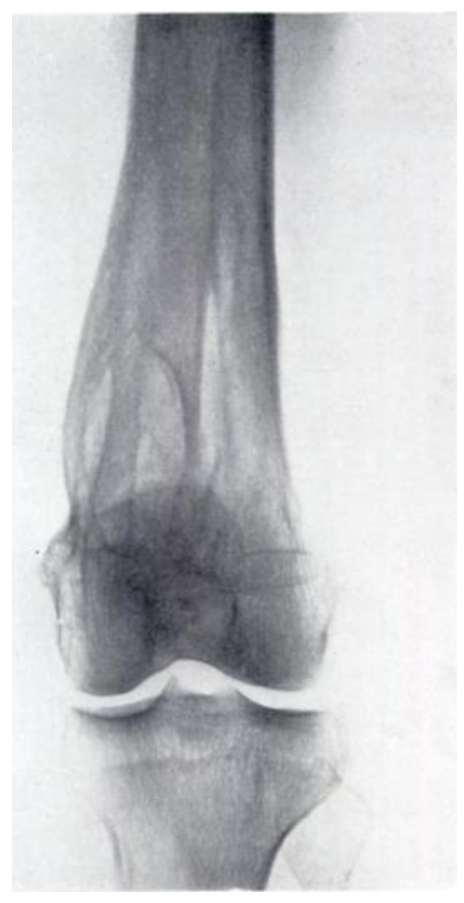

Fig. 3

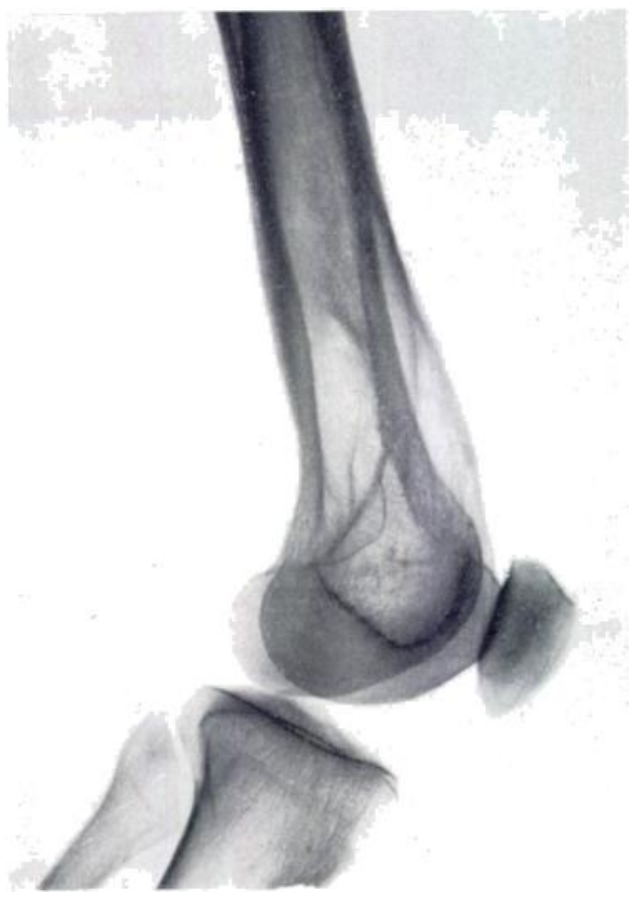

Fili. 4

Case 1. Figure 3 . Intero-posterior radiograph of lower end of left femur, showing several areas of increased translucency. The largest of these is a longitudinally disposed ellipse which has bulged the medial cortex. Its poles are connected by a band of bone of normal densits: Lateral to it is a wine-bottle shaped area. Notice the early osteoarthritic lipping on the medial side. Figure 4-lateral radiograph of lower end of left femur, showing two streaks of increased density merging into normal bone proximally and distally.

The blood count, serum calcium, serum phosphorus and serum alkaline phosphatase, were normal. The radiographic changes (Figs. 2 to 4 ) were confined to the left hip and femur, and were therefore monomelic. The hip showed malformation and the femur contained several areas of increased translucency. Early osteoarthritic changes were present in the left knee.

After a two months' course of short-wave diathermy, quadriceps exercises and a single injection of ten millilitres of $\mathbf{2}$ per cent procaine into the nodules in the left groin, the patient had negligible symptoms.

Case $2-A$ forty-year-old man had pain round his left ear and cheek; this was associated with a lower motor neurone lesion of the left facial nerve due to geniculate herpes, from which he recovered completely within five weeks. He also suffered from neurofibromatosis, without disability. No other member of his family was affected.

On examination the skin changes were like those in the first case. His left tibia was $4 \cdot 5$ centimetres longer than his right, but his femora were the same length. There was marked forward bowing of his left tibia (Fig. 5). The front of its lower half was covered with a firm, lobulated swelling tightly stretching the skin without adhering to it. The swelling was 18 centimetres long, 9 centimetres wide and up to 5 centimetres

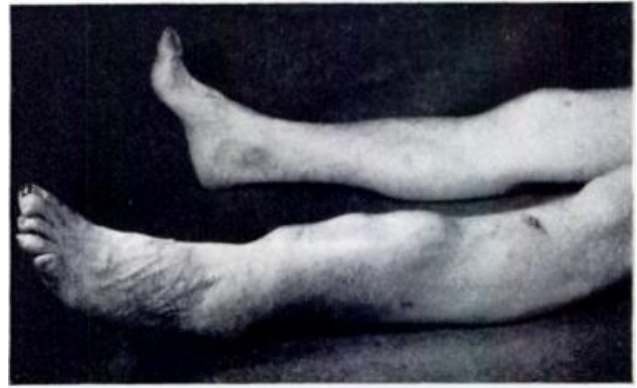

FIG. 5

Case 2-Photograph demonstrating the forward bowing of the left tibia and the soft-tissue swelling covering it.

vol. 34 B, No. 1, FEBRUARY 1952 


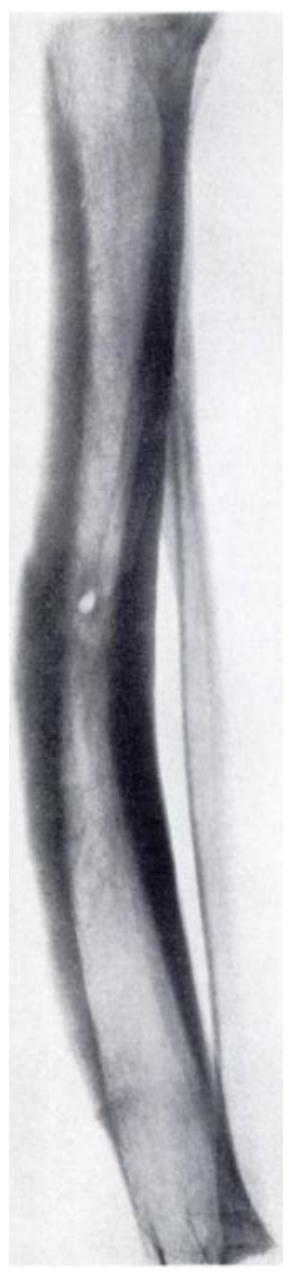

FIG. 6

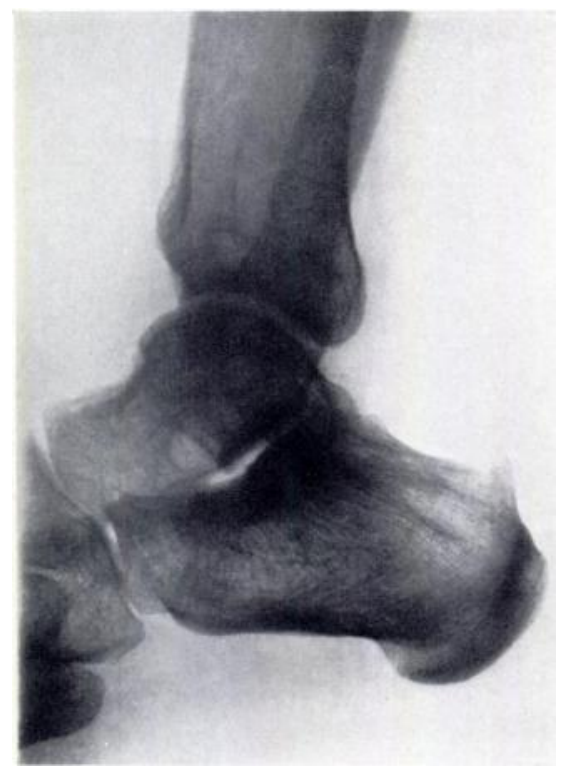

FIG. 7

Case 2. Figure 6-Lateral radiograph of the left tibia, showing cortical sclerosis and thickening and anterior roughening. Figure 7-Lateral radiograph of the left ankle, showing early osteoarthritic lipping at the ankle and talo-navicular joints and roughening of the upper border of the talus.

deep. Its margins blended with the tibia. His left foot and ankle were clinically normal. He dipped to the right when he walked, and he had a compensatory lumbar scoliosis.

The radiographic changes, again monomelic, were confined to the left tibia and talus. The tibia showed forward bowing, cortical sclerosis and thickening (especially anteriorly) and a radiolucent area in the medullary carity just below its midpoint (Fig. 6). Early osteoarthritic lipping was present in the ankle and talonavicular joints. The dorsal surface of the talus appeared roughened (Fig. 7).

\section{DISCUSSION}

About one-third of the reported cases of skeletal neurofibromatosis have joint changes, which fall into three main groups: 1) dysplasias; 2) sclerosis of the articulating bone end; and 3) secondary osteoarthritis.

Dysplasias have been recorded thus:

At the hip-Coxa valga (Brooks and Lehman 1924), coxa valga associated with a shallow acetabulum as in Figure 2, coxa vara (Uhlmann and Grossman 1940), congenital dislocation (Holt and Wright 1948).

At the foot and ankle-Calcaneus deformity associated with distortion, underdevelopment and displacement of all the tarsal bones (Moore 1941), congenital rocker foot (same author), 
congenital flat foot due to misshapen tarsal bones (same author), lateral displacement of talus and calcaneus (Brooks and Lehman 1924).

In the hand-Hypertrophy of the bones of the first and second digital rays associated with expanded bone ends (McCarroll 1950).

Sclerosis of the articulating bone ends, though often accompanying the dysplasias, has been observed as the chief abnormality at the following joints:

At the hip-Sclerosis of the acetabulum associated with several radiolucent areas (Friedman 1944).

At the ankle-Sclerosis of lower part of tibia and talus (Holt and Wright 1948).

At the wrist-Sclerosis at distal end of radius, and sclerosis and hypertrophy of the carpal bones (McCarroll 1950).

Secondary osteoarthritis may occur in:

1) Abnormal joints, e.g., the coxa valga described by Fairbank (1950).

2) Normally developed joints subjected to abnormal forces, e.g., a short femur causing rocking of the knee with every step as in Case 1, or forward bowing of the tibia producing abnormal compression of the ankle as in Case 2.

Incomplete though this list of joint changes may be, it is nevertheless noteworthy that a fairly thorough search of the literature has revealed no record of shoulder or elbow lesions in patients suffering from neurofibromatosis.

\section{SUMMARY}

1. Two patients with monomelic bone and joint changes in neurofibromatosis have been described.

2. The joint changes in neurofibromatosis include dysplasias, sclerosis of the articulating bone ends and secondary osteoarthritis.

\section{REFERENCES}

Brooks, B., and Lehman, E. P. (1924): The Bone Changes in Recklinghausen's Neurofibromatosis. Surgery, Gynecology and Obstetrics, 38, 587.

Fairbank, H. A. T. (1950): Neurofibromatosis. Journal of Bone and Joint Surgery, 32-B, 266.

Friedmax, M. M. (1944): Neurofibromatosis of Bone. American Journal of Roentgenology and Radium Therapy, 51, 623.

Gould, E. P. (1918): The Bone Changes occurring in von Recklinghausen's Disease. Quarterly Journal of Medicine, 11, 221.

Holt, J. F., and Wright, E. M. (1948): The Radiologic Features of Neurofibromatosis. Radiology, $51,647$. McCarroll, H. R. (1950): Clinical Manifestations of Congenital Neurofibromatosis. Journal of Bone and Joint Surgery, 32-A, 601.

Moore, B. H. (1941): Some Orthopaedic Relationships of Neurofibromatosis. Journal of Bone and Joint Surgery, 23, 109.

Uhlmans, E., and Grossman, A. (1940): Von Recklinghausen's Neurofibromatosis with Bone Manifestations. Annals of Internal Medicine, 14, 225. 